\title{
Leopold Landau †
}

Wieder ist einer der Führer unserer gynäkologischen Wissenschaft dahingegangen. Am 28. Dezember 1920 ist Leopold Landau nach kurzer Krankheit gestorben. Noch vor zwei Jahren konnten wir seinen 70. Geburtstag feiern, den er in der gleichen körperlichen und geistigen Frische beging, die wir von ihm seit seinem erst en Auftreten in Berlin als junger Privatdozent der Gynäkologie und Geburtshilfe gewöhnt $\Lambda$ varen. Als Schüler Spiegelbergs habilitierte er sich 1872 in Breslau und siedelte von dort 1876 nach Berlin über. Landau war immer eine Kampfnatur, und was er einmal für richtig und für die Wissenschaft fördernd erkannt hatte, das vertrat er, auch wenn es gegen die gerade herrschende Schule war. Als ein vorzüglich durchgebildeter allgemeiner Arzt und mit ausgezeichnetem ärztlichen Blick bekämpfte er die Aus- $\Lambda$ vüchse der operativen Gynäkologie, die sich zu einer Zeit geltend machten, als unsere Spezialwissenschaft begann, sich eine selbständige Stellung zu erringen. Ich erinnere hier an seine Arbeiten über Ovarie, die er in Gemeinschaft mit Remak als Teilerscheinung der Hysterie richtig erkannte und darum gegen die damals als Heilung empfohlene Exstirpation der Ovarien mit aller Eneigie-Front machte. Die spätere und namentlich die neueste Zeit, in welcher wir die Bedeutung der inneren Sekretion kennengelernt haben, haben ihm nur zu sehr recht gegeben. Heute erscheint uns dies selbstverständlich, aber wer, wie ich, die damaligen Kämpfe und die gehässige Art, mit der er von

128 Leopold Landau $\dagger$. anderer Seite angegriffen wurde, mitgemacht hat, der weiß, daß es nur die lautersten Motive waren, welche ihn zu seinen polemischen Schriften veranlaßten, urn der Wahrheit zum Nutzen der Kranken zum Siege zu verhelfen. So ging es auch mit seiner kleinen, aber hoch-bedeutsamen Schrift über Gynäkologíschen Spezialismus (1884), in der er sich gegen diejenigen wendet, die in der Gynäkologie nichts als die Behandlung der weiblichen Genitalien sahen. Es ist ihm wahrlich nicht leicht gemacht worden, und wenn er sich trotzdem eine führende Stellung erobert hat, so hat er dies seinem genialen Blick für das prinzipiell Wiehtige, seiner glänzenden Rednergabe und seiner un-ermüdlichen Arbeitskraft zu danken. An alien grundlegenden Fragen hat er in hervorragender Weise teilgenommen und durch seine hervor-ragende kritische Begabung verstanden, das wirklich Gute der All-gemeinheit nutzbar zu machen. Und wenn man bedenkt, wie schwer es ihm wurde, in privater Tätigkeit, ohne das Material einer Universitätsklinik oder eines Krankenhauses, alles dies aus sich heraus zu schaffen, so ist sein» Verdienst nur um so höher einzuschätzen. Aus ganz kleinen Anfängen heraus ist sein mustergültiges klinisches und poliklinisches Institut entstanden, dem von jeher ein pathologischmikroskopisches Laboratorium angegliedert war, denn er hatte schon frühzeitig erkannt, welche Bedeutung die mikroskopische Untersuchung für viele gynäkologische Erkrankungen hat. Jetzt bei seinem Tode wurde von hervorragender pathologischer Seite noch besonders hervorgehoben, welch eine Fülle von Anregungen und Unterstützungen die pathologische Anatomie dem Material der Landa uschen Klinik ver-dankt. Von ganz grundlegender Bedeutung für die damalige Zeit war seine Arbeit über die Erweiterung der Gebärmutter (1887), in der bei Aborten und sonstigen intrauterinen Erkrankungen die digitale Aus-stattung des Uterus gefordert wurde, 
um den sich häufenden Per-forationen bei Auskratzung des Uterus ohne vorherige Erweiterung ein Ziel zu setzen. In seiner Pathologie und Therapie der Beckenabszesse des Weíbes, mit besonderer Berücksichtigung der vaginalen Radikal-operation, hat er dieser letzteren Operation zuerst die Wege in Deutsch-land geebnet. Seine Arbeiten über Wanderníere, Wanderleber und Hängebauch der Frauen, über subdiaphragmatische Echinokokken, über Echinokokken an und in der weiblichen Brust, über intermittierende Hydronephrose und Myxödem zeigen, wie er sich jederzeit bemühte, die Beschwerden der Frauen auf ihre wahre Ursache zurückzuführen und beweisen seinen vorzüglichen allgemein medizinischen Blick. Im Jahre 1893 wurde er Titularprofessor, um einige Jahre später zum Geheimen Medizinalrat ernannt zu werden.. Als sehr beliebter akademischer Lehrer hat er im Laufe der Jahre eine große Reihe von Schülern heran-gebildet, die seine Lehren in die ganze Welt hinausgetragen haben. Trotz seiner großen praktischen und wissenschaftlichen Tätigkeit hatte er aber von Anfang an immer die Zeit gefunden, sich an den sozialen Bestrebungen der Ãrzte zu beteiligen, war Mitglied der Ärztekammer und stellvertretender Vorsitzender der Berliner medizinischen Gesell-schaft, deren Ehrenmitglied er in Anerkennung der Verdienste wurde, welche er sich um das

Zustandekommen des Langenbeck-Virchow-

Leopold Landau $\uparrow$.

129

Hauses erworben hatte. 16 Jahre lang war er Mitglied der Stadt-verordnetenversammlung in Berlin und hat besonders bei der Ein-richtung der Infektionsabteilung im Rudolf VirchowKrankenhaus und bei der Schaffung des Medizinalamtes der Stadt Berlin hervorragend mitgearbeitet. Während des Weltkrieges war er noch Chefar $3 / 4$ t eines Reservelazarettes und veröffentlichte hier eine sehr interessante Mit-teilung über einen Fall von Schußverletzung der Leber.

Ein reiches, arbeitsames Leben hat mit dem Tode Leopold Landaus seinen Abschluß gefunden. Aber was er geschaffen hat, wird in unserer Wissenschaft für alle Zeiten bleibend sein. Karl Abel-Berlin.

Ârbeiten Leopold Landaus.

1873: Zur Physiologie der Bauchspeichelabsonderung. Diss. Breslau.1874: Über Melaena der Neugeborenen. - 1874: Die operative Gynäkologie mit Einschluß der gynäkologischen Untersuchungslehre. Kritische Besprechung. - 1877: Vorschläge zum Leichenschaugesetz, das Puerperal fieber betreffend. Berl. klin. Woch. - 1880: Zur operativen Behandlung der Echinokokken in der Bauchhöhle. Berl. klin. Woch. - 1881: Die Wanderniere der Frauen. Berlin, August Hirschwald. - 1882: Zur Verhütung des Puerperalfiebers. Dtsch. med. Zeit. - 1882: Zur Technik der Laparotomien. Zbl. f. Gyn. - 1882: Über die Anwendung der Glühhitze in der Gynäkologie. Dtsch. med. Zeitg. - 1882: Zur Operation der Echino kokken in der Bauchhöhle. Zbl. f. Gyn. - 1884: Gynäkologischer Spezialismus. Dtsch. med. Zeitg. - 1885: Über Dislokation der Leber. Dtsch. med. Woch. 1885: Ein Fall von Ovariotomie bei hysterischer Hemianästhesie. Klinischer Beitrag zur Ovarie- und Kastrationsfrage. Zusammen mit Remak. Ztschr. f. klin. Med. Bd. 6. - 1885: Über die Bedeutung des sogenannten Ovarial- oder Iliakalschmerzes bei Hysterischen. Dtsch. med. Woch. - 1885: Zur Operation der Echinokokken der Bauchhöhle, besonders der Leber. Langenbecks Archiv. -- 1885: Über den diagnostischen Wert 
der Rectaluntersuchung mit der vollen Hand in gynäkologischer Beziehung. Arch. f. Gyn. Bd. 7. - 1885: Zur Kasuistik der Echinokokken an und in der weiblichen Brust. Arch. f. Gyn. Bd. 8. - 1885: Erwiderung an Hegar und Kaltenbach. Arch. f. Gyn. Bd. 9. - 1885: Über Entstehung, Erkenntnis und Behandlung der Harnleiterscheidenfisteln. Arch. f. Gyn. Bd. 9. - 1885: Über Indikation und Wert der künstlichen Frühgeburt bei engem Becken. Arch. f. Gyn. Bd. 11. - 1885: Zur Lehre von der Eierstocksschwangerschaft. Arch. f. Gyn. Bd. 16. - 1885: Über Selbstinfektion im Wochenbette. Arch. f. Gan. Bd. 23. - 1885: Die Wander leber und der Hängebauch der Frauen. Berlin, Hirschwald. - 1887: Zur Erweiterung der Gebärmutter. Dtsch. Med. Ztg. - 1887: Über Myxödema. Berl. klin. Woch. - 1888: Über intermittierende Hydronephrose. Berl. klin. Woch. - 1890: Über Schwangerschaft im Nebenhorn. Berl. klin. Woch. 1890: Klinische Beobachtung und mikroskopische Diagnose. Zbl. f. Gyn. 1890: Zur mikroskopischen Diagnose des Gebärmutterkrebses. Zbl. f. Gyn. - 1890: Therapeutische Erfahrungen über Tubensäcke (Hydrosalpinx, Pyosalpinx). 10. internat. med. Kongreß. - 1891: Über Tubensäcke Eine klinische Studie. Berlin. Hirschwald. - 1894: Pathologie und Therapie bei Beckeneiterung. Zeitschr. f. Geb. und Gyn. -- 1894: Zur Pathologie Monatssehi·ift f. Geburtshíufe u. Gynäkologie. Bd. LIV. Heft 2. 9 130

Leopold Landau $\uparrow$.

und Therapie der Beekenabszesse des Weibes mit besonderer Berücksichtigung der vaginalen Radikaloperation. Arch. f. Gyn. - 1894: Über die Heilimg der Beckenabszesse des Weibes mit besonderer Berücksichtigung der vaginalen Radikaloperation. Berl. med. Gesellsch. - 1895: Tabellarisehe Zusamnienstellung der von L. Landau von 1893-1895 ausgeführten vaginalen Radikaloperationen bei chromsch-eitrigen, resp. entzündlichen Erkrankungen der Gebärmutteranhänge. Berlin. Als Manuskript gedruckt bei L. Schumacher. - 1895: Über Klemmbehandlung bei Karzinoma und Myoma uteri, bei Pyosalpinx und Beckeneiterungen. Verhdlg. der dtsch. Ges. f. Gyn. - 1895: Bericht über 109 Fälle von vaginaler Radikaloperation bei doppelseitigen chronisch eitrigen resp. entzündlichen Adnexerkran-kungen. Berl. klin. Woch. - 1896: Die vaginale Radikaloperation. Technik und Geschichte. Zusammen mit Th. Landau. Berlin, August Hirschwald. Ins Englische übersetzt von Eastman und Giles. London 1897. 1896: Be-merkungen zu Herrn Sängers Vortrag: Zu $\gamma$ Doyenschen Methode der Hysterectomis vaginalis. Zbl. f. Gyn. - 1896: Zur Behandlung von Be-schwerden der natürlichen und antizipierten Klimax mit Eierstockssubstanz. Berl. klin. Woch. - 1897: Wanderniere und Unfall. Ärztl. Sachverst.-Ztg.

1897: Entgegnung an Herrn Sänger. Zbl. f. Gyn. - 1897: Zur Technik der abdominalen Totalexstirpation des Uterus. Zbl. f. Gyn. -- 1899: Anatomische und klinische Beiträge zur Lehre von den Myomen am weib-lichen Sexualapparat. Berlin und Wien. Urban \& Schwarzenberg. - 1899: Beitrag zur Myomlehre mit Demonstrationen. Verhdlg. d. dtsch. Ges. f. Gyn. - 1899: Wann muß ein Myom operiert werden ? Therapie der Gegenwart. - 1899: Zur Symptomatologie und Therapie der Uterus-myome. Berl. klin. Woch. - 1900: Zwei Todesfälle nach Ausschabung der Gebärmutter. Fahrlässige Tötung ? Ärztl. Sachverst.-Ztg. - 1900: Diskussion zu dem Vortrag des Herrn F. König: Die chirurgische Behandlung der Nierentuberkulose. Dtsch. med. Woch. - 1900: Nierenausschaltung durch 
Harnleiterunterbmdung. Dtsch. med. Woch. - 1900: Über den frühen Nachweis von freier Bauchwassersucht. Zbl. f. Gyn. - 1901: Symptome und Behandlung cler Myome. Dtsch. Klin. 1901: Über Behandlung der Bauchwassersucht. Med. Woche. - 1901: Über eine bisher nicht bekannte Form des Gebärmutterverschlusses. Berl. klin. Woch. - 1901: Einige Bemerkungen zur Behandlung der Extrauterinschwangerschaft. Dtsch. med. Presse. - 1901: Fahrlässige Tötung nach Darmzerreißung bei vermeintlicher Lösung der Nachgeburt. Ärztl. Sachverst. Ztg. - 1902: Rein mobile. Pathogenie, Symptomes es Indications opératoires. Französ. Ges. f. L L1/8ologie. Paris. - 1904: Zum klinischen Verhalten des Epithelioma chorioektoderrnale. Berl. klin. Woch. 1905: Erste Hilfe bei Blutungen aus dem weiblichen Genitaltraktus. Ztschr. f. ärztl. Fortbild. 1908: Über duodenalen Ileus nach Operationen. Berl. klin. Woch. - 1910: Wandlungen und Fortschritte in der Behandlung der chronisch-entzündlichen und eitrigen Erkrankungen der Gebärmutteranhänge. Berl. klin. Woch. - 1915: Kurze Mitteilung über einen Fall von Schußverletzung der Leber. Ztschr. f. ärztl. Fortbild. - 1915: Cholarkos nach Schuß durch die Leber. Berl. klin. Woch. -- Außerdem sehr zahlreiche Diskxissionsreden. die hier nicht im einzelnen aufgeführt werden konnten. 